\title{
Improving Direct Counting for Frequent Itemset Mining
}

\author{
Adriana Prado ${ }^{\star}$, Cristiane Targa ${ }^{\star \star}$, and Alexandre Plastino ${ }^{\star \star \star}$ \\ Department of Computer Science, Universidade Federal Fluminense, \\ Rua Passo da Pátria, 156 - Bloco E - $3^{\circ}$ andar - Boa Viagem, \\ 24210-240, Niterói, RJ, Brazil \\ \{aprado, ctarga, plastino\}@ic.uff.br \\ http://www.ic.uff.br
}

\begin{abstract}
During the last ten years, many algorithms have been proposed to mine frequent itemsets. In order to fairly evaluate their behavior, the IEEE/ICDM Workshop on Frequent Itemset Mining Implementations (FIMI'03) has been recently organized. According to its analysis, $\mathrm{kDCl}++$ is a state-of-the-art algorithm. However, it can be observed from the FIMI'03 experiments that its efficient behavior does not occur for low minimum supports, specially on sparse databases. Aiming at improving $\mathrm{kDCl}++$ and making it even more competitive, we present the $\mathrm{kDCl}-3$ algorithm. This proposal directly accesses candidates not only in the first iterations but specially in the third one, which represents, in general, the highest computational cost of $\mathrm{kDCl}++$ for low minimum supports. Results have shown that $\mathrm{kDCl}-3$ outperforms $\mathrm{kDCl}++$ in the conducted experiments. When compared to other important algorithms, kDCl-3 enlarged the number of times $\mathrm{kDCl}++$ presented the best behavior.
\end{abstract}

\section{Introduction}

Association rules represent an important type of information extracted from data mining processes that describe interesting relationships among data items of a specific knowledge domain. Market basket analysis is the typical application of association rule mining $(A R M)$ and consists in identifying relationships among products that significantly occur in customers buys. For instance, the rule "a customer who buys bean, in general buys rice", represented by $\{$ bean $\} \Rightarrow\{$ rice $\}$, would certainly be extracted from a market database in Brazil. Formally, an association rule, defined over a set of items $\mathcal{I}=\left\{i_{1}, i_{2}, \ldots, i_{n}\right\}$, is an implication of the form $X \Rightarrow Y$, where $X \subset \mathcal{I}, Y \subset \mathcal{I}, X \neq \varnothing, Y \neq \varnothing$, and $X \cap Y=\varnothing$. We say that $X$ is the antecedent and $Y$ is the consequent of the rule.

Let $\mathcal{D}$ be a set of transactions (a transactional database) defined over $\mathcal{I}$, where each transaction $t$ is a subset of $\mathcal{I}(t \subseteq \mathcal{I})$. Then, the rule $X \Rightarrow Y$ holds in

\footnotetext{
* Work sponsored by CAPES Master scholarship.

$\star \star$ Work sponsored by CAPES Master scholarship.

$\star \star \star$ Work sponsored by CNPq research grant 300879/00-8.
} 
$\mathcal{D}$ with support $s$ and confidence $c$ if, respectively, $s \%$ of the transactions in $\mathcal{D}$ contain $X \cup Y$, and $c \%$ of the transactions in $\mathcal{D}$ that contain $X$ also contain $Y$.

The $A R M$ problem is commonly broken into two phases. Let minsup and minconf be, respectively, the user specified minimum support and confidence. The first phase, the frequent itemset mining (FIM) phase, consists in identifying all frequent itemsets (sets of items) that occur in at least minsup $\%$ of the transactions. The second phase outputs, for each identified frequent itemset $Z$, all association rules $A \Rightarrow B$ with confidence greater than or equal to minconf, such that $A \subset Z, B \subset Z$, and $A \cup B=Z$. The FIM phase demands more computational effort than the second one and has been intensively addressed [3].

\subsection{Previous Work}

During the last ten years, many algorithms have been proposed to efficiently mine frequent itemsets. Most of them are improved variations of Apriori [1]. In this strategy, the set $F_{1}$ containing all frequent itemsets of length 1 (1-itemsets) is initially identified. Then, at each iteration $k \geq 2$ : (a) the set $C_{k}$ of candidates of length $k$ ( $k$-candidates) is generated by combining all pairs included in $F_{k-1}$ (frequent $(k-1)$-itemsets) that share a common $(k-2)$-prefix; (b) $C_{k}$ is pruned in order to eliminate candidates that have at least one $(k-1)$-subset that is not frequent (all subsets of a frequent itemset are also frequent); (c) then, in the counting phase, the database $\mathcal{D}$ is read and, for each transaction $t$ of $\mathcal{D}$, the support of all $k$-candidates contained in $t$ is incremented. After reading the whole database, $F_{k}$ is identified. If this set is empty, the termination condition is reached. In the Apriori algorithm, the candidates are stored in a hash-tree.

Subsequent proposed algorithms have improved Apriori by reducing: the number of database scans $[11,12]$, the computational cost of the counting phase $[2$, 7-9], and the number and size of transactions to be scanned [7-9].

Another class of FIM algorithms mines frequent itemsets by adopting a depth-first approach. The Eclat algorithm [14] uses an in-memory vertical layout of the database and an intersection-based approach to determine the supports of the candidates. The FP-growth algorithm [5] does not generate candidates. It builds a compact in-memory representation of the database, called FP-tree (frequent pattern tree), from which the support of all frequent itemsets are derived. According to experiments presented in [5], FP-growth is very efficient. However, it did not show good performance on sparse databases [15].

OpportuneProject [6], PatriciaMine [10] and FPgrowth* [4] are recent FIM algorithms that have improved the ideas adopted by FP-growth. OpportuneProject is able to choose between two different data structures according to the database features. PatriciaMine uses only one data structure (Patricia trie) to represent both dense and sparse databases together with optimizations that reduce the cost of tree traversal and that of physical database projections. FPgrowth* introduces an array-based technique also aiming at reducing FP-trees traversals.

DCl (Direct Count 8 Intersect) [8] and its recent version $\mathrm{kDCl}++[7]$ are apriori-like algorithms. During their first iterations, they exploit database pruning techniques, inspired in [9], and efficient data structures to store candidates. 
When the vertical layout of the pruned database fits into main memory, the supports of the candidates are obtained by an intersection-based technique. Both of them can adapt their behavior according to the database features. Moreover, $\mathrm{kDCl}++$ uses a counting inference strategy based on that presented in [2].

A recent and efficient proposed method called LCM-freq [13] mines all frequent itemsets from frequent closed itemsets (an itemset is a closed itemset if none of its supersets have the same support).

\subsection{Motivation}

As pointed out in [3], every new proposed FIM algorithm is many times evaluated by limited experimental tests. In order to fairly evaluate the behavior of these algorithms, the IEEE/ICDM Workshop on Frequent Itemset Mining Implementation (FIMI'O3) has been recently organized. All the accepted FIM implementations can now be found in the FIMI repository (available at http://fimi.cs.helsinki.fi/) together with an extensive performance evaluation.

According to the FIMI'03 Workshop experiments, $\mathrm{kDCl}++$ is a state-of-theart algorithm. However, it can be observed from its analysis [3] that its highly efficient behavior is not true for low values of support, specially on sparse databases.

Aiming at evaluating $\mathrm{kDCl}++$ under these specific circumstances, we performed experiments on different combinations of databases and minimum supports also used in $[2-8,10,13,15]$. We observed that the third iteration presented a very high computational cost compared to the other iterations.

Table 1 presents, for different databases and low minimum supports, the total execution times (in seconds) of $\mathrm{kDCl}++$ and its third iteration execution times (in seconds). The databases are described in Section 4. The last column represents the ratio between the third iteration execution time and the total execution time. We observed that, on average, the third iteration represented $65 \%$ (ranged from $34 \%$ to $83 \%$ ) of the total execution time of $\mathrm{kDCl}++$ runs. This is due to the huge number of 3-candidates that must be evaluated. Indeed, as already observed in $[7,8]$, the third iteration may represent a bottleneck in apriori-like algorithms.

In this work, in order to improve the $\mathrm{kDCl}++$ algorithm, we propose a strategy, called $\mathrm{kDCl}-3$, that enables the direct counting at the third iteration, one of its most time consuming iterations for low minimum supports, specially on sparse databases. This proposal is based on a directly accessible data structure to store candidates, which allows a more efficient 3-candidate counting.

The paper is organized as follows. In Section 2, we review the $\mathrm{kDCl}++$ algorithm. In Section 3, we present the $\mathrm{kDCl}-3$ algorithm. The experimental results are reported and discussed in Section 4. Finally, in Section 5, some concluding remarks are made and future work is pointed out. 
Table 1. Execution times of $\mathrm{kDCl}++$

\begin{tabular}{|l|c|c|c|}
\hline Database (minsup - \%) & Total time & $3^{\text {rd }}$ iteration time & $(\%)$ \\
\hline T20I10N1KP5C0.25D200K (0.1) & 544 & 353 & 65 \\
T20I10N1KP5C0.25D200K (0.3) & 51 & 34 & 67 \\
T10I5N1KP5C0.25D200K (0.01) & 298 & 220 & 74 \\
T10I5N1KP5C0.25D200K (0.03) & 104 & 70 & 67 \\
T30I15N1KP5C0.25D200K (0.25) & 655 & 541 & 83 \\
T30I15N1KP5C0.25D200K (0.5) & 139 & 116 & 83 \\
T25I10D10K (0.1) & 26 & 21 & 81 \\
T25I10D10K (0.2) & 4 & 1.9 & 47 \\
T40I10D100K (0.5) & 386 & 271 & 70 \\
T40I10D100K (0.75) & 133 & 101 & 76 \\
T10I4D100K (0.01) & 307 & 103 & 34 \\
T10I4D100K (0.03) & 36 & 28 & 78 \\
T30I16D400K (0.4) & 775 & 487 & 63 \\
T30I16D400K (0.6) & 234 & 161 & 69 \\
BMS-POS (0.1) & 422 & 156 & 37 \\
BMS-POS (0.3) & 47 & 22 & 47 \\
\hline
\end{tabular}

\section{The $\mathrm{kDCl}++$ Algorithm}

The $\mathrm{kDCl}++$ algorithm [7] is an apriori-like strategy. During its first iterations, it exploits directly accessible data structures together with database pruning techniques that reduce the number and size of transactions to be scanned.

For $k=2, \mathrm{kDCl}++$ builds a prefix table $P_{2}$ of $\left(\begin{array}{c}\left|F_{1}\right| \\ 2\end{array}\right)$ entries. Each entry of $P_{2}$ is a counter that represents a 2-candidate and accumulates its support.

To directly access the entry (counter) associated with a generic candidate $c=\left(c_{1}, c_{2}\right)$, where $c_{1}<c_{2}, \mathrm{kDCl}++$ maps $c$ into a pair $\left\{x_{1}, x_{2}\right\}$ where $x_{1}=\mathcal{T}\left(c_{1}\right)$, $x_{2}=\mathcal{T}\left(c_{2}\right)$, and $\mathcal{T}$ is a strictly monotonous increasing function defined by $\mathcal{T}$ : $F_{1} \rightarrow\left\{1, \ldots,\left|F_{1}\right|\right\}$. Equation 1 is thus adopted by $\mathrm{kDCl}++$ in order to find the entry of $P_{2}$ that represents $\mathrm{c}=\left\{c_{1}, c_{2}\right\}$, called here $E P_{2}\left(c_{1}, c_{2}\right)$. This equation is derived considering that the counters associated with pairs $\left\{1, x_{2}\right\}, 2 \leq x_{2} \leq\left|F_{1}\right|$ are stored in the first $\left(\left|F_{1}\right|-1\right)$ positions of $P_{2}$, the counters associated with pairs $\left\{2, x_{2}\right\}, 3 \leq x_{2} \leq\left|F_{1}\right|$, are stored in the next $\left(\left|F_{1}\right|-2\right)$ positions, and so on.

$$
E P_{2}\left(c_{1}, c_{2}\right)=\sum_{i=1}^{x_{1}-1}\left(\left|F_{1}\right|-i\right)+\left(x_{2}-x_{1}\right)=\left|F_{1}\right|\left(x_{1}-1\right)-\frac{x_{1}\left(x_{1}-1\right)}{2}+x_{2}-x_{1}
$$

At the end of the counting phase, if the corresponding entry of $c$ is greater than or equal to minsup, $c$ is included in $F_{2}$.

When $k>2$, another data structure was proposed. A prefix table $P_{k}$ of $\left(\begin{array}{c}\left|M_{k}\right| \\ 2\end{array}\right)$ entries is built, where $M_{k}$ is the set of items at iteration $k$ that were not pruned during execution progress. Each entry of $P_{k}$ contains a pointer to the 
beginning of a memory section that stores the ordered $k$-candidates having the same 2-prefix. The entry of $P_{k}$ that represents the prefix $\left\{c_{1}, c_{2}\right\}$ of a candidate $c=\left\{c_{1}, c_{2}, \ldots, c_{k}\right\}$ is obtained similarly to the second iteration.

To obtain the support of each $k$-candidate, for each transaction $t, \mathrm{kDCl}++$ determines all possible 2-prefixes of all $k$-itemsets in $t$. Then, for each 2-prefix, the entry $i$ of $P_{k}$ is obtained and the section of ordered candidates that must be evaluated will be delimited by $P_{k}[i]$ and $P_{k}[i+1]$.

At each iteration $k \geq 2, \mathrm{kDCl}++$ checks whether the vertical layout of the pruned database fits into main memory. The vertical layout can be seen as a set of $\left|M_{k}\right|$ bit vectors of size $\left|T_{k}\right|$, where $T_{k}$ is the set of not pruned transactions at that iteration. If the bit vector associated with an item $i$ has its $j^{\text {th }}$ bit equal to 1 , item $i$ is present in the $j^{\text {th }}$ transaction. If the database is small enough, its vertical representation is built and the supports of the $(k+1)$-candidates is thus obtained by the intersections of the $k$ bit vectors associated with their items.

\section{The kDCl-3 Algorithm}

Aiming at improving the performance of $\mathrm{kDCl}++$, in this section, we propose the $\mathrm{kDCl}-3$ algorithm, which uses an efficient direct counting technique to determine the support of all 3-candidates.

The data structure used by $\mathrm{kDCl}-3$ during the third iteration is based on the prefix table $P_{2}$ and on a new array $C$ which represents all 3-candidates.

In the $\mathrm{kDCl}-3$ algorithm, an entry of $P_{2}$ related to the frequent 2-itemset $\left\{c_{1}, c_{2}\right\}$ stores two values. The first one, represented by $\operatorname{Pt}\left(c_{1}, c_{2}\right)$, is a pointer to the first entry of the contiguous section in $C$ associated with all 3-candidates (in lexicographic order) having the same 2-prefix $\left\{c_{1}, c_{2}\right\}$. This value is null if there is no 3 -candidate with that 2-prefix. The second value is the order, represented by $\operatorname{Ord}\left(c_{1}, c_{2}\right)$, in which the frequent 2-itemset $\left\{c_{1}, c_{2}\right\}$ is represented in $P_{2}$. $\operatorname{Ord}\left(c_{1}, c_{2}\right)$ is null if $\left\{c_{1}, c_{2}\right\}$ is not a frequent 2 -itemset. To access $P_{2}, \mathrm{kDCl}-3$ utilizes Equation 1, similarly to $\mathrm{kDCl}++$.

The entry of $C$ which corresponds to a generic 3-candidate $c=\left\{c_{1}, c_{2}, c_{3}\right\}$, called here $E C\left(c_{1}, c_{2}, c_{3}\right)$ and defined by Equation 2, can be found from the pointer $\operatorname{Pt}\left(c_{1}, c_{2}\right)$ together with an offset value. This offset is defined by the number of frequent 2-itemsets represented between $\left\{c_{1}, c_{2}\right\}$ and $\left\{c_{1}, c_{3}\right\}$ in $P_{2}$.

$$
E C\left(c_{1}, c_{2}, c_{3}\right)=\operatorname{Pt}\left(c_{1}, c_{2}\right)+\left(\left(\operatorname{Ord}\left(c_{1}, c_{3}\right)-\operatorname{Ord}\left(c_{1}, c_{2}\right)\right)-1\right) .
$$

Figure 1 illustrates how $\mathrm{kDCl}-3$ identifies the entry associated with a given 3 -candidate. In this example, $F_{1}=\{0,1,3,7,9\}$ and $F_{2}=\{\{0,1\},\{0,3\},\{0,7\},\{0,9\}$, $\{1,3\},\{1,9\},\{3,9\}\}$. The set of 3 -candidates $\left(C_{3}\right)$, represented by $C$, is generated in lexicographic order by the combination of the frequent 2-itemsets that share a common 1-prefix. For instance, the frequent 2 -itemset $\{0,1\}$ will be combined with all subsequent frequent 2 -itemsets in $P_{2}$ that have 0 as their first item, in order to generate all 3 -candidates with $\{0,1\}$ as their 2-prefix. Since the frequent 2 -itemset $\{0,9\}$ is the third one after $\{0,1\}$, it is easy to conclude that the candidate $\{0,1,9\}$ is the third candidate in $C$ having $\{0,1\}$ as their 2-prefix. Then, the 
3 -candidate $\{0,1,9\}$, generated by $\{0,1\}$ and $\{0,9\}$, is located in $C$ two entries after the first 3 -candidate sharing the prefix $\{0,1\}$. Indeed, this two entries are given by $((\operatorname{Ord}(0,9)-\operatorname{Ord}(0,1))-1)$ and the first 3 -candidate sharing the prefix $\{0,1\}$ is identified by $\operatorname{Pt}(0,1)$.

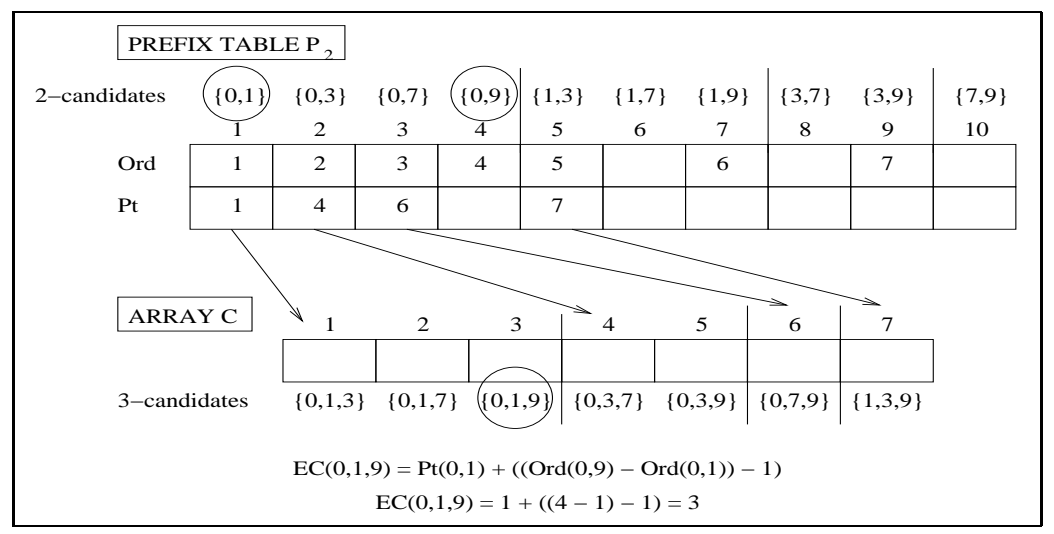

Fig. 1. Data structure used by kDCl-3 to access 3-candidates

To obtain the support of each 3-candidate, for each transaction $t, \mathrm{kDCl}-$ 3 determines all the possible 3 -itemsets included in $t$. After that, for each 3itemset $\left\{c_{1}, c_{2}, c_{3}\right\}$ in $t$, it identifies the entries of $P_{2}$ that represents the 2 -itemsets $\left\{c_{1}, c_{2}\right\}$ and $\left\{c_{1}, c_{3}\right\}$. If the identified entries represent frequent 2-itemsets, the entry associated with $\left\{c_{1}, c_{2}, c_{3}\right\}$, in $C$, is found from Equation 2 and is then incremented. At the end of the candidate counting, if an entry in $C$ is greater than or equal to minsup, its corresponding candidate is included in $F_{3}$.

\section{Performance Evaluation}

The computational experiments reported in this work have been carried out on a $600 \mathrm{MHz}$ Pentium III PC with $256 \mathrm{MB}$ of RAM memory under the RedHat Linux 9.0 (kernel version 2.4.20) operating system.

The databases used in the experiments are described in Table 2. The first three databases were generated by the IBM dataset generator [1]. The databases T40I10100K, T10I4D100K and BMS-POS (real database) were downloaded from the FIMI repository, and finally, T25I10D10K and T30I16D400K were downloaded from the $\mathrm{DCl}$ site (http://miles.cnuce.cnr.it/ ${ }^{\sim}$ palmeri/datam/DCI).

This section is organized as follows. In Subsection 4.1, we compare the effectiveness of the new technique used by $\mathrm{kDCl}-3$ with that adopted by $\mathrm{kDCl}++$ during the third iteration. In Subsection 4.2, we compare the performance of kDCl-3 with recent FIM algorithms evaluated in the FIMI'03 Workshop. 
Table 2. Databases used in the experiments and its corresponding characteristics

\begin{tabular}{|l|c|c|c|c|}
\hline Database & Items & Transactions & Avg. Length & References \\
\hline T20I10N1KP5C0.25D200K & 1000 & 197,437 & 20.1 & {$[3]$} \\
T10I5N1KP5C0.25D200K & 1000 & 192,889 & 10.3 & {$[3]$} \\
T30I15N1KP5C0.25D200K & 1000 & 199,093 & 29.6 & {$[3]$} \\
T25I10D10K & 1001 & 9,219 & 27.7 & {$[2,5,7,8]$} \\
T40I10D100K & 1000 & 100,000 & 39.6 & {$[4,10,13]$} \\
T10I4D100K & 1000 & 100,000 & 10.1 & {$[10,13]$} \\
T30I16D400K & 1000 & 397,487 & 29.7 & {$[7,8,10]$} \\
BMS-POS & 1657 & 515,597 & 6.5 & {$[3,6,10,13,15]$} \\
\hline
\end{tabular}

\subsection{Effectiveness of the New Direct Counting Technique}

For $\mathrm{kDCl}++$, we used the source code available at the $\mathrm{DCl}$ site, now also available at the FIMI repository. For $\mathrm{kDCl}-3$, we adapted the source code of $\mathrm{kDCl}++$ in order to implement $\mathrm{kDCl}-3$ features and to allow a fair comparison between them.

Table 3 shows, for the same combinations of databases and low minimum supports presented in Table 1, the total execution times (in seconds) and the third iteration execution times (in seconds) of $\mathrm{kDCl}++$ and $\mathrm{kDCl}-3$, respectively. The fourth column represents the ratio between the third iteration execution time of $\mathrm{kDCl}-3$ and that of $\mathrm{kDCl}++$. The seventh column represents the ratio between the total execution time of $\mathrm{kDCl}-3$ and that of $\mathrm{kDCl}++$.

Table 3. Execution times of $\mathrm{kDCl}++$ and $\mathrm{kDCl}-3$

\begin{tabular}{|c|c|c|c|c|c|c|}
\hline & \multicolumn{3}{|c|}{$3^{\text {rd }}$ iteration times } & \multicolumn{3}{|c|}{ Total times } \\
\hline Database (minsup - \%) & $\mathrm{kDCl}++$ & kDCl-3 & $(\%)$ & $\mathrm{kDCl}++$ & $\mathrm{kDCl}-3$ & $(\%)$ \\
\hline T20I10N1KP5C0.25D200K (0.1) & 353 & 40 & 11 & 544 & 191 & 35 \\
\hline T20I10N1KP5C0.25D200K (0.3) & 34 & 10 & 29 & 51 & 27 & 53 \\
\hline T10I5N1KP5C0.25D200K (0.01) & 220 & 12 & 5 & 298 & 90 & 30 \\
\hline T10I5N1KP5C0.25D200K (0.03) & 70 & 7 & 10 & 104 & 48 & 46 \\
\hline T30I15N1KP5C0.25D200K (0.25) & 541 & 86 & 16 & 655 & 209 & 32 \\
\hline T30I15N1KP5C0.25D200K (0.5) & 116 & 31 & 27 & 139 & 60 & 43 \\
\hline T25I10D10K (0.1) & 21 & 4 & 19 & 26 & 9 & 35 \\
\hline T25I10D10K (0.2) & 1.9 & 1.3 & 68 & 4 & 3.7 & 93 \\
\hline T40I10D100K (0.5) & 271 & 69 & 25 & 386 & 208 & 54 \\
\hline T40I10D100K (0.75) & 101 & 39 & 39 & 133 & 72 & 54 \\
\hline T10I4D100K (0.01) & 103 & 6 & 6 & 307 & 202 & 66 \\
\hline T10I4D100K (0.03) & 28 & 3 & 11 & 36 & 16 & 44 \\
\hline T30I16D400K (0.4) & 487 & 132 & 27 & 755 & 478 & 63 \\
\hline T30I16D400K (0.6) & 161 & 67 & 42 & 234 & 154 & 66 \\
\hline BMS-POS (0.1) & 156 & 9 & 6 & 422 & 216 & 51 \\
\hline BMS-POS (0.3) & 22 & 5 & 23 & 47 & 25 & 53 \\
\hline
\end{tabular}


Due to the large number of 3 -candidates generated in these tests (up to $11,051,970$ on $\mathrm{T} 10 \mathrm{I} 4 \mathrm{D} 100 \mathrm{~K}(0.01 \%)), \mathrm{kDCl}++$ presented a poor performance in the third iteration. We can note that $\mathrm{kDCl}-3$ efficiently reduced the execution time of this time consuming phase. We can observe, for instance, that in the first line of Table 3, while $\mathrm{kDCl}++$ executed the third iteration in 353 seconds, $\mathrm{kDCl}-3$ executed it in 40 seconds. This represents a reduction of $89 \%$. Since the third iteration represents in this run a great part of the total execution time (as observed in Subsection 1.2), kDCl-3 total execution time was 35\% of that of $\mathrm{kDCl}++$ (a reduction of $65 \%$ ).

The elapsed times of $\mathrm{kDCl}-3$ were, for the third iteration, on average, $23 \%$ (from $5 \%$ to $68 \%$ ) of that of $\mathrm{kDCl}++$ (a reduction, on average, of $77 \%$ ). We can also observe the impact produced by the proposed direct counting technique during the third iteration in the total execution times. The relative total execution times of $\mathrm{kDCl}-3$ ranged from $30 \%$ to $66 \%$ (with a discrepant value of $93 \%$ ) representing, on average, $51 \%$ of the total execution times of $\mathrm{kDCl}++$.

\subsection{Performance Comparison}

This section reports a performance comparison among $\mathrm{kDCl}-3$ and four preeminent algorithms: $\mathrm{kDCl}++$, PatriciaMine, FPgrowth*, and LCM-freq. According to [3], $\mathrm{kDCl}++$ and PatriciaMine are considered state-of-the-art algorithms. We also selected FPgrowth* and LCM-freq since they showed a good behavior in the FIMI'03 experiments, specially for low supports. For PatriciaMine, FPgrowth* and LCM-freq, we used the source codes available at the FIMI repository.

Figure 2 shows the relative execution times of $\mathrm{kDCl}-3$ and the other algorithms. In each picture, the value 1 (in $y$-axis) represents the relative execution time of the worst algorithm for the correspondent minimum support. The value in brackets beside the minimum support (in $x$-axis) is the absolute execution time of the worst algorithm (in seconds).

We observe that $\mathrm{kDCl}-3$ was better than $\mathrm{kDCl}++$ for almost all combinations of databases and minimum supports. In graph (e), for the highest support (0.9\%), and in graph (f) for the three highest supports $(0.5 \%, 0.4 \%$, and $0.3 \%), \mathrm{kDCl}++$ was slightly better than $\mathrm{kDCl}-3$ due to a not relevant number of 3-candidates.

For lower values of minimum support in graphs (e) and (f), when the number of 3-candidates increases, $\mathrm{kDCl}-3$ performs much better than $\mathrm{kDCl}++$. In some cases, as pointed out in [7], the bad behavior of $\mathrm{kDCl}++$ can be justified by the size of $C_{3}$, which can lead to "a lot of useless work to determine the support of many candidate itemsets which are not frequent".

In graphs $(\mathrm{g})$ and $(\mathrm{h}), \mathrm{kDCl}++$ was always the worst algorithm. For all values of support, $\mathrm{kDCl}-3$ was better than $\mathrm{kDCl}++$, making it more competitive.

In graphs (a)-(d), we observe that for higher minimum supports, $\mathrm{kDCl}-3$ runs faster than $\mathrm{kDCl}++$, which was the best algorithm in these situations. For lower minimum supports, however, $\mathrm{kDCl}++$ performance decreases, but $\mathrm{kDCl}-3$ is still better than $\mathrm{kDCl}++$, also making it more competitive. In these graphs (and also in $\operatorname{graph}(\mathrm{e})$ ), for some values of support, $\mathrm{kDCl}++$ was the worst algorithm (or almost the worst), while the improvements of $\mathrm{kDCl}-3$ made it the best one. 

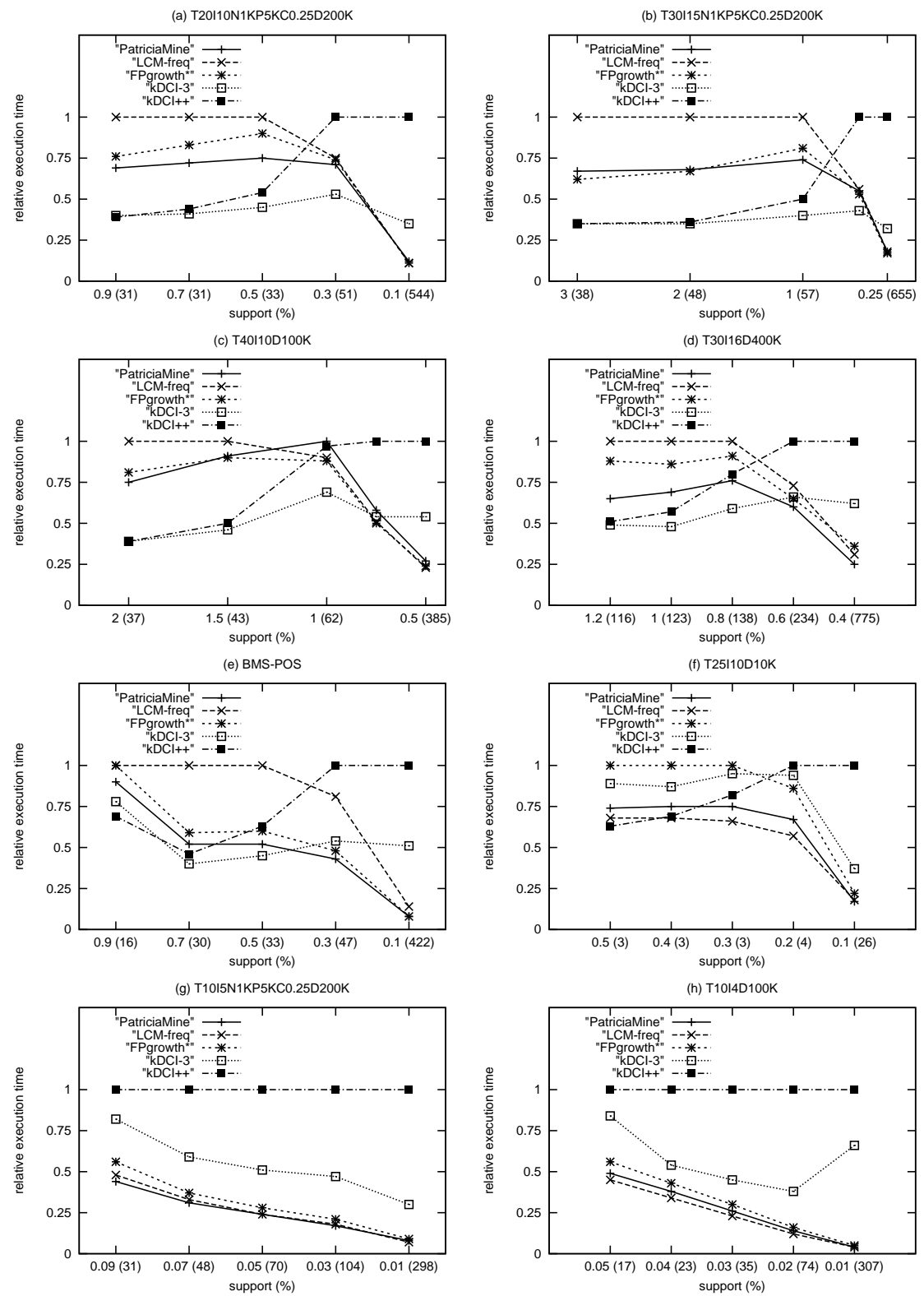

Fig. 2. Relative execution times of $\mathrm{kDCl}-3$ and other algorithms 


\section{Conclusions}

In this work we proposed $\mathrm{kDCl}-3$, an extension of the $\mathrm{kDCl}++$ algorithm - a recent and important method for the FIM problem. $\mathrm{kDCl}-3$ provided an efficient direct counting of candidates when dealing with a huge number of 3-candidates, a consequence of low minimum support values, specially in sparse databases.

From the conducted computational experiments, we observed that $\mathrm{kDCl}-3$ presented a very efficient behavior. $\mathrm{kDCl}-3$ reduced the execution times of the third iteration of $\mathrm{kDCl}++$ and, consequently, its total elapsed times in almost all executed experiments. We believe that these results represent an important contribution that improves a state-of-the-art algorithm.

Based on the encouraging observed results, as future work, we intend to investigate the use of the proposed direct counting of candidates in other iterations.

\section{References}

1. R. Agrawal and R. Srikant. Fast Algorithms for Mining Association Rules. In 20th VLDB Conference, 1994.

2. Y. Bastide, R. Taouil, N. Pasquier, G. Stumme, and L.Lakhal. Mining Frequent Patterns with Counting Inference. In ACM SIGKDD Explorations, v.2, n.2, 2000.

3. B. Goethals and M. J. Zaki. Advances in Frequent Itemset Mining Implementations: Introduction to FIMI'03. In IEEE ICDM FIMI Workshop, 2003.

4. G. Grahne, J. Zhu. Efficiently Using Prefix Trees in Mining Frequent Itemsets. In IEEE ICDM FIMI Workshop, 2003.

5. J. Han, J. Pei, and Y. Yin. Mining Frequent Patterns without Candidate Generation. In ACM SIGMOD Conference, 2000.

6. J. Liu, Y. Pan, K. Wang, and J. Han. Mining Frequent Item Sets by Opportunistic Projection. In 8th ACM SIGKDD Conference, 2002.

7. S. Orlando, P. Palmerimi, R. Perego, C. Lucchese, and F. Silvestri. kDCI++: A Multi-Strategy Algorithm for Discovering Frequent Sets in Large Databases. In IEEE ICDM FIMI Workshop, 2003.

8. S. Orlando, P. Palmerimi, and R. Perego. Adaptive and Resource-Aware Mining of Frequent Sets. In IEEE ICDM Conference, 2002.

9. J. S. Park, M. Chen, and P. S. Yu. An Effective Hash-Based Algorithm for Mining Association Rules. In ACM SIGMOD Conference, 1995.

10. A. Pietracaprina and D. Zandolin. Mining Frequent Itemsets using Patricia Tries. In IEEE ICDM FIMI Workshop, 2003.

11. A. Savasere, E. Omiecinski, and S. Navathe. An Efficient Algorithm for Mining Association Rules in Large Databases. In 21th VLDB Conference, 1995.

12. H. Toivonen. Sampling Large Databases for Association Rules. In 22th VLDB Conference, 1996.

13. T. Uno, T. Asai, Y. Uchida, and H. Arimura. LCM: An Efficient Algorithm for Enumerating Frequent Closed Item Sets. In IEEE ICDM FIMI Workshop, 2003.

14. M. J. Zaki, S. Parthasarathy, M. Ogihara, and W. Li. New Algorithms for Fast Discovery of Association Rules. In 3rd ACM SIGKDD Conference, 1997.

15. Z. Zheng, R. Kohavi, and L. Mason. Real World Performance of Association Rule Algorithms. In 7th ACM SIGKDD Conference, 2001. 